\title{
Simulation Study of Fluid Flow and Estimation of a Heterogeneous Porous Media Properties Using Lattice Gas Automata Method
}

\author{
Dedy Kristanto $^{1 *}$ and Windyanesha Paradhita ${ }^{2)}$ \\ 1) Petroleum Engineering Department, Faculty of Mineral Technology, UPN "Veteran" Yogyakarta \\ 2) Magister Student of Petroleum Engineering Department, UPN "Veteran" Yogyakarta \\ * corresponding email: dedikristanto@upnyk.ac.id
}

\begin{abstract}
Most models used in reservoir simulation studies are on the scale of meters to hundreds of meters. However, increasing resolution in geological measurements results in finer geological models. Simulations study of particle movements provide an alternative to conventional reservoir simulation by allowing the study of microscopic and/or macroscopic fluid flow, which is close to the scale of geological models. In this paper, the FHP-II (Frisch, Hasslacher and Pomeau FHP) model of lattice gas automata were developed to study fluid flow in order to estimate the properties of heterogeneous porous media. Heterogeneity simulated by placing solid obstacles randomly in a two-dimensional test volume. Properties of the heterogeneous porous media were estimated by the shape, size, number of the obstacles and by the distribution of the obstacles within the volume. Results of the effects of grain sizes and shapes, and its distribution in the porous media on the tortuosity, effective porosity, permeability and displacement efficiency were obtained. An investigation of fluid flow and comparison with laboratory experiment were also presented. Reasonably good agreement between the lattice gas automata simulation and laboratory experiment results were achieved.
\end{abstract}

Keywords: heterogeneity, lattice gas automata, porous media, simulation

\section{INTRODUCTION}

Modeling of fluid flow in porous media for both single-phase and two-phase flows is of importance in petroleum engineering. Most models for reservoir simulations are on the scale of centimeter to hundreds of meters. Usually, increasing resolution in geological measurements result in finer geological models. Many numerical methods have been developed to simulate fluid flow in porous media. Numerical models of fluid flow in porous media can be developed from either microscopic or macroscopic properties. Attention is then typically focused on the determination of the petrophysical properties of the porous media and its performance based on the microscopic pore-space geometry. Due to the intrinsic inhomogeneity of porous media makes the application of proper boundary conditions difficult. Hence, microscopic flow calculations have typically been achieved with idealized arrays of geometrically simple pores and throats.

Because fluid flow in porous media is an important subject in petroleum engineering, numerous theoretical and experimental studies have attempted to investigate its performance. Rothman (1988), reported that although these investigations are diverse in approach, they can be classified broadly into three categories based on their use of microscopic data. First, some studies employ no microscopic data at all; these studies attempt instead to relate macroscopic rock properties, such as relating permeability to resistivity and porosity (e.g., Walsh and Brace, 1984; Paterson, 1983). In the second category are studies that collect microscopic data on pore-space geometry, usually via microscopic and digital image analysis (e.g., Lin and Cohen, 1982), and then compute macroscopic statistics from these microscopic data in attempt to relate their macroscopic rock properties to the statistical properties (e.g., Berryman and Blair, 1986; Lin et al., 1986). The third category is based entirely on microscopic rock geometry (e.g., Koplik et al., 1986).

The finite difference and finite element methods have been useful for simulating single-phase and two-phase flow in porous media, and have been used extensively. Numerical methods based on the finite difference approximation of the governing equations are probably the most commonly used tools for simulating the single-phase and two-phase flow process, and predicting their performance. In practice, the porous media are usually represented by discrete grid block, and transfer of each constituent being tracked is computed across each block face for a succession of small-time increments. Finite difference or finite element methods use floating-point numbers to describe properties, a large number of grid blocks are often required, and appropriate boundary conditions are difficult to be applied. As a result, they may not be the most efficient numerical method for this problem. 
Despite this extensive study, Dullien (1979), shown that theoretical estimates of macroscopic rock properties are often in error by as much as an order of magnitude or more. The lack of success of these theoretical models, however, could be the result of faulty flow models, inadequate representations of pore space, or both. In this research, a different approach is used to model the fluid flow in heterogeneous porous media. The difference between this work and the existing theoretical literature (finite element and/or finite difference methods) on fluid flow in porous media lies in the numerical method used to model the fluid flow. The numerical method used is Lattice Gas Automata. This alternative method was first introduced by Hardy et al. (1973; 1976) and was extended by Frisch et al. (1986; 1987), for the computational of fluid dynamic. The method is based on the knowledge of microscopic rock geometry, which falls in the third category detailed above. This is due to the microgeometric space as the Navier-Stokes equations are solved numerically with appropriate boundary conditions. Therefore, the results obtained with the lattice gas automata should agree well with the experimental results insofar as the microscopic model adequately represents the real porous media. Hence, lattice gas automata methods are applicable to the study of fluid flow in porous media.

\section{METHODS}

\subsection{Lattice Gas Automata}

In 1986, Frisch, Hasslacher and Pomeau introduced a lattice gas model based on a hexagonal grid (FHP-models). In the FHP-models each of the particles travels with unit speed and an exclusion principle is applied allowing only one particle to travel in each direction along a link. Rest particles can also be introduced into the model. A rest particle remains at rest at a site, link, but is able to take part in a collision with particles arriving at the site. The six link directions can be seen in Figure 1, and the velocity vectors $\left(c_{i}\right)$ is given by Equation 1.

$$
c_{i}=\left(\cos \frac{\pi i}{3}, \sin \frac{\pi i}{3}\right), \quad i=1,2, \ldots, 6
$$

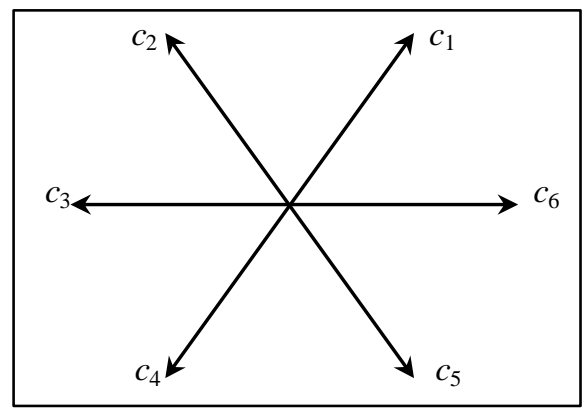

Figure 1. The hexagonal lattice used in the FHP models

Source: Frisch et al., 1986

The simplest of the FHP models is the FHP-I in which there are no rest particles and gives a total of 5 collisions out of the 64 possible in-states. The second is FHP-II model that introduces a rest particle and allows the particles to collide according to the rules that six rest particle creation collisions. This model gives 22 possible interactions out of a possible total of 128. The third is FHP-III model that extensions of FHP-II, which allow all collision conserve mass and momentum at each site and give 76 possible collisions.

At the start of each time step the particles at each site collide according to the particular collision rules for the model being used - the collision stage. After the collision stages each particle travels in a straight line along one of the lattice links, unless it is a rest particle, until it arrives at the next link - the propagation (streaming) stage. The particles arriving at their new sites then collide at the beginning of the next time step. In the FHP models, each link of the hexagonal lattice may carry one particle (of the given mass/momentum/energy state) at any one time, this called exclusion principles. The particles of fluid site on a hexagonal lattice can be seen in Figure 2.

Figure 2, shows that each arrow represents a particle of unit mass moving in the direction given by the arrow. The lattice is initially prepared so that no more than one particle is moving with a particular velocity at a particular site on the lattice Figure 2a. Then, the particles hop and scatter can be seen in Figure 2b; each particle moves one lattice unit in the direction of its velocity. Scattering can be seen in Figure 2c; if two or more particles arrive at the same site, they can collide. Some collisions cause the particles to scatter that is their velocity change. In all cases, however, collisions may change neither the total number of particles nor the vector sum of the velocities. The only collisions that have changed the configuration of particles are located in the middle row. In other words, mass and momentum are conserved. The particle dynamics depicted in Figure 2 expressed by the equation, 


$$
n_{i}\left(x+c_{i}, t+1\right)=n_{i}(x, t)+\Delta_{i}[\mathrm{n}(x, t)]
$$

Here the time $t$ is integer-valued and the duration of a time step is taken to be unity. The quantities $\mathrm{n}=\left(n_{1}, n_{2}, \ldots, n_{6}\right)$ are Boolean variables that indicate the presence $\left(n_{i}=1\right)$ or absence $\left(n_{i}=0\right)$ or particles moving from a lattice site situated at position $x$ to the neighboring site situated at position $x+c_{i}$, where the particles move with unit speed in the directions given by Equation 1. The function $\Delta_{i}$ is called the collision operator. Its describes the change in $n_{i}(x, t)$ due to collisions, and takes on the values 0,1 and -1 . It is the sum of quantities that may be written as Boolean or logical, expressions, one for each possible collision.

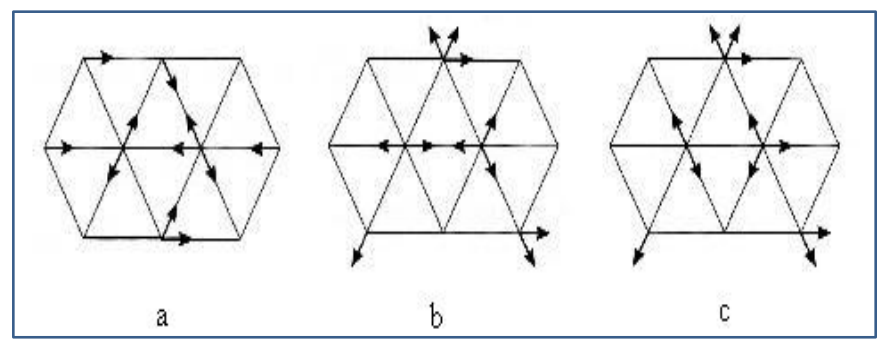

Figure 2. One-time step in the evolution of the FHP models: (a) initial condition; (b) propagation step of the particles; (c) result of collisions step of the particles Source: Rothman et al., 1997

There are three types of FHP model of lattice gas automata, i.e., FHP-I, FHP-II, and FHP-III, which have been developed, based on the particle's usage (with or without rest particles) and the possible outcomes collision configurations occurred (Frisch et al., 1986; Rothman et al., 1997). The simplest of the FHP models is the FHP-I in which there are no rest particles, and gives 64 possible in-states. The FHP-II model introduces a rest particle and allows the particles to collide according to the following rules, and this gives 128 possible interactions outcomes. While, the FHP-III model is an extension of FHP-II, which allows all collisions conserve mass and momentum at each site, and gives 76 possible collisions. This work used the FHP-II model of lattice gas automata to study microscale heterogeneity of a porous media.

Zanetti (1989), defined a lattice-gas automata as a particle of gas that occupy the site of a regular lattice and can hop from the lattice sites to the nearest neighbors. Lee et al. (1993), used the lattice gas automata method for hydrodynamic calculations. The lattice gas automata method employs interactions of discrete fluids on a regular lattice analogous to microscopic molecular dynamics. Therefore, a complex system can be simulated by simple rules of particle interactions at a lattice. Macroscopic variables are then recovered by averaging over a spatial and temporal space. Computationally this method has two main advantages over conventional methods. Firstly, the mathematical operations are mainly bit manipulation, which provides memory efficiency, thereby easily simulating a very large system. Secondly, the algorithm is inherently parallel. While, Orme (1996), showed that the lattice gas automata model has been devised to assist in understanding the properties of real fluid and it could provide an alternative to computational fluid dynamic analysis for predicting fluid flow.

\subsection{Simulation Model}

In the FHP-II model of lattice gas automata used, each site of two-dimensional hexagonal lattice particle can move into any of six directions. The particles of unit mass and unit velocity moving along the lattice links of unit length connecting the node to its six nearest neighbors is expressed by Equation 1 of Frisch et al. (1986) and Rothman et al. (1997). At a given time $t_{n}$, the state of the lattice $x_{i j}$ is defined by,

$$
n\left(x_{i j}, t_{n}\right)=\left\{n_{i}\left(x_{i j}, t_{n}\right)\right\} i=1,2, \ldots, 6
$$

where $n_{i}\left(x_{i j}, t_{n}\right)$ is a Boolean variable; $n_{i}\left(x_{i j}, t_{n}\right)=1$ if the $i$-th cell is occupied and 0 if it is vacant. Evolution of the system is specified by the collision and propagation rules for particles can be seen in Figure 2. One-particle distribution function at each node of the 6-bit state, $f_{i}(x, t)$ gives the probability of finding a particle with velocity $c_{i}$ at position $x$ and time $t$. Furthermore, updating of $f$ includes two processes, i.e., collision is given by Equation 4, and propagation is given by Equation 5, respectively, where $\Delta_{i}$ signify the particle velocity change due to collision.

$$
\begin{aligned}
& f_{i}(x, t)=f_{i}(x, t)+\Delta_{i}(f(x)) \\
& f_{i}(x, t)=f\left(x+c_{i}, t+1\right)
\end{aligned}
$$


The collision phase of each time step is the process of transformation between the input and output states of the lattice sites under a set of collision rules. Collision rules are expressions by Boolean variable, which define the relationship between the input and output states of a site and updated according to Equation 6.

$$
n_{i}\left(x_{i j}, t_{n}^{+}\right)=n_{i}\left(x_{i j}, t_{n}^{-}\right)+\Delta_{i}\left\{n\left(x_{i j}, t_{n}^{-}\right)\right\}
$$

where $\Delta_{i}$ is the collision function which takes the value of 1 or 0 , and $\xi$ is the random variable $(\xi=1)$. Here $t_{n}^{-}$and $t_{n}^{+}$ stand for the time of pre- and post-collision. The microscopic Boolean states of all the particles are averaged to obtain density and velocity using equations,

$$
\begin{aligned}
& \rho=\sum_{i=0}^{6} N_{i}\left(x_{i j}, t_{n}\right) \\
& \rho \mathrm{u}=\sum_{i=0}^{6} c_{i} N_{i}\left(x_{i j}, t_{n}\right)
\end{aligned}
$$

The pressure is given by,

$$
p=\frac{3}{7} \rho
$$

While, viscosity of fluid is given by equation of d'Humieres et al. (1986),

$$
v=\frac{1}{28} \frac{1}{d(1-d)^{3}} \frac{1}{1-4 d / 7}-\frac{1}{8}
$$

where $d$ is the mean density per link $(=\rho / 7)$. Viscosity is a function of the collision rules used and the density of the fluid particles.

The permeability coefficient $(k)$, which a measure of fluid conductivity through the porous media in this simulation is given by Carman-Kozeny equation, which includ the factors of effective porosity and tortuosity,

$$
k=\frac{\phi_{e f f}{ }^{3}}{c \tau^{2} S^{2}}
$$

where $c$ is the Kozeny coefficient. The effective porosity $\left(\phi_{e f f}\right)$ of the porous media is given by equation,

$$
\phi_{\text {eff }}=a x^{3}-\left(2 a+\phi_{C}\right) x^{2}+\left(a+1-\phi_{C}\right) x
$$

where

$$
x=\frac{\left(\phi-\phi_{C}\right)}{\left(1-\phi_{C}\right)}
$$

and $a$ is constant $(a=0.3)$, and $\phi_{C}$ is a critical porosity or percolation threshold, respectively. Tortuosity $(\tau)$ of the porous media is determined using equation,

$$
\tau=0.8(1-\phi)+1
$$

and specific surface area $(S)$ is determined by equation,

$$
S=-\frac{z}{R_{0}} \phi \ln \phi
$$

where $z$ is two-dimensional space $(z=2)$, and $R_{0}$ is the hydraulic radius of the obstacles. Furthermore, the displacement efficiency $\left(E_{D}\right)$ is predicted by equation,

$$
E_{D}=\frac{N_{p}}{V_{p}} \times 100 \%
$$

where $N_{p}$ is the oil recovery obtained at the end of the displacement process, and the pore volume $\left(V_{p}\right)$ of porous media is calculated by equation, 


$$
V_{p}=V_{b} \phi_{e f f}
$$

where $\phi_{\text {eff }}$ is an effective porosity as given by Equation 12 .

\section{RESULTS AND DISCUSSION}

\subsection{Constructing Fluid Flow in a Heterogeneous Porous Media}

The simulation to construct a fluid flow in heterogeneous porous media was conducted on the $800 x 600$ lattice units. A method of constructing a model of heterogeneous porous media is to place solid obstacles randomly in a twodimensional test volume. Black dots indicate solid obstacles formed. The obstacles can have irregular shapes caused by overlapping of the obstacles. The fluid was forced to move from the left side to the right side of the porous media model by applying a force on the particles. Density of fluid particles per lattice site was 1.54 to 2.25 , which provides a good approximation with Frisch (1986), where the range was from 1.5 to 3.5 particles per lattice site. Velocity field it its position is displayed by black arrows. At each location, the length of the arrow represents the amplitude of the velocity and the direction of the arrow points to the direction of the flow. Visualization for each arrow was an average of $10 x 10$ lattice sites.

The simulation results to construct a heterogeneous porous media by spherical and rectangular shapes for each grain size can be seen in Figure 3 and Figure 4. It shows that the pore configuration is complex, but the pores are relatively uniformly distributed. Complex pore configurations arise from the interaction of packing (relates with the grains shape arrangement) and grain size distribution factors of the framework fraction. System pore openings of the interconnected pores with small pore opening and larger pore opening to represent the porosity and permeability of porous media may occur in such porous media constructed. The streamline of the fluid flow through porous media to distinguish the direction of the flow was also observed.

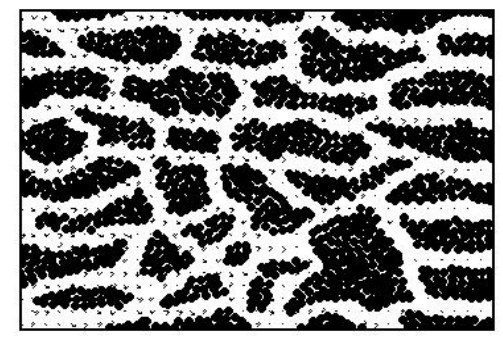

(a) Grain size 10 lu

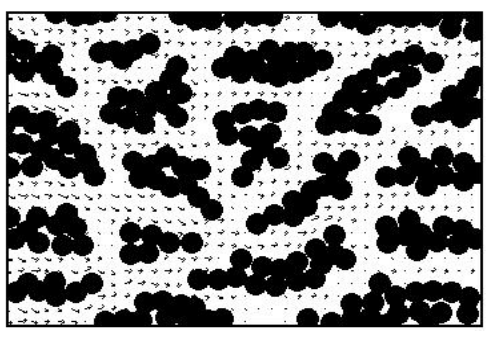

(b) Grain size 20 lu

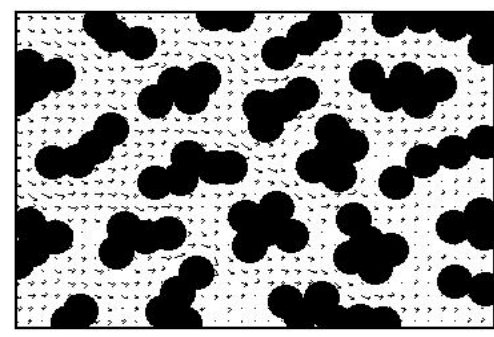

(c) Grain size 30 lu

Figure 3. The example of fluid flow simulated in heterogeneous porous media of spherical obstacles with porosity $20 \%$ for different grain sizes at 8000 -time steps

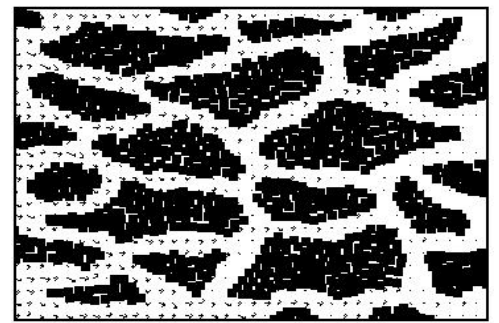

(a) Grain size $10 \mathrm{lu}$

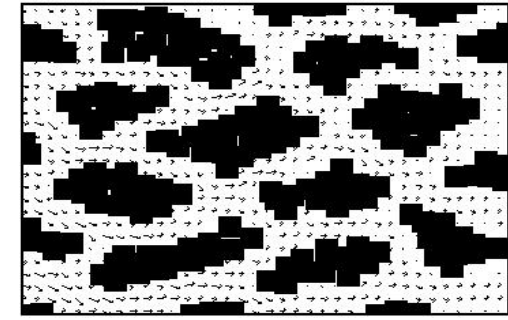

(b) Grain size 20 lu

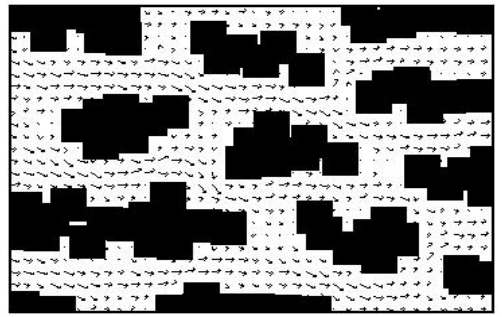

(c) Grain size 30 lu

Figure 4. The example of fluid flow simulated in heterogeneous porous media of rectangular obstacles with porosity $20 \%$ for different grain sizes at 8000-time steps

The majority of the flow follow several winding paths in general can be seen in Figure 3 and Figure 4. However, there are some dead regions where flow is very slow. The flow velocity increases at the locations where the size of pores decreases. The velocity field in the figures also shows that the flow tends to pass through from the dead regions and continuously flow through the outlet (right side) of the porous media. Furthermore, Figure 3 and Figure 4 also shown that the physical models of heterogeneities that can be constructed by this simulation are heterogeneous isotropic distribution of permeability, and anisotropic distribution of heterogeneous permeability, as proposed by Gao (1994). 
Anisotropic implies that the variation of porosity and permeability values of porous media are different in $x$ and $y$ directions.

Based on the simulation results, indicate that the complex geometry of heterogeneous porous media was successfully formed by FHP-II model of the lattice gas automata. The complex geometry of heterogeneous porous media can be defined from the porosity point of view. Complex geometry is that type of porous media with heterogeneous porosity, which depends on its solid particle shape and size. The representation obtained from this simulation is promising and shows an attractive achievement, since results of the simulation seem to predict the system's critical parameter, i.e., permeability for different types of porous media with respect to their properties. The prediction shows that the simulation applied has a wide range of applicability for different geometry shapes. Furthermore, the porous media and porosity constructed are dependent on the distribution of grain size and shape, where the porosity increases as the grain sizes increases. The distribution of grain size and shape also affects on the packing arrangements of solid obstacles and velocity field of the fluid flow.

\subsection{Properties of a Heterogeneous Porous Media}

As a physical quantity, the most intuitive and straightforward definition of tortuosity is the ratio of the average length of true flow paths to the length of the system in the direction of macroscopic flux. By this definition, tortuosity depends not only on the microscopic geometry of the pores, but also on the transport mechanism under consideration. The comparison of simulated tortuosity for spherical and rectangular obstacles can be seen in Figure 5.

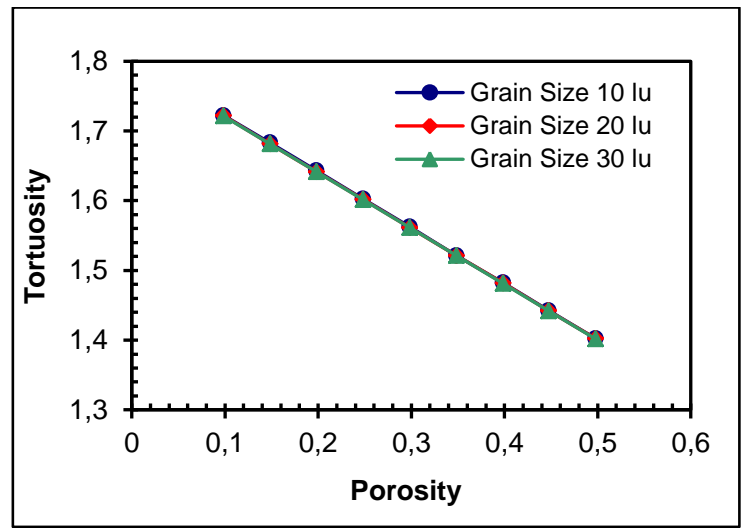

(a) Spherical Obstacles

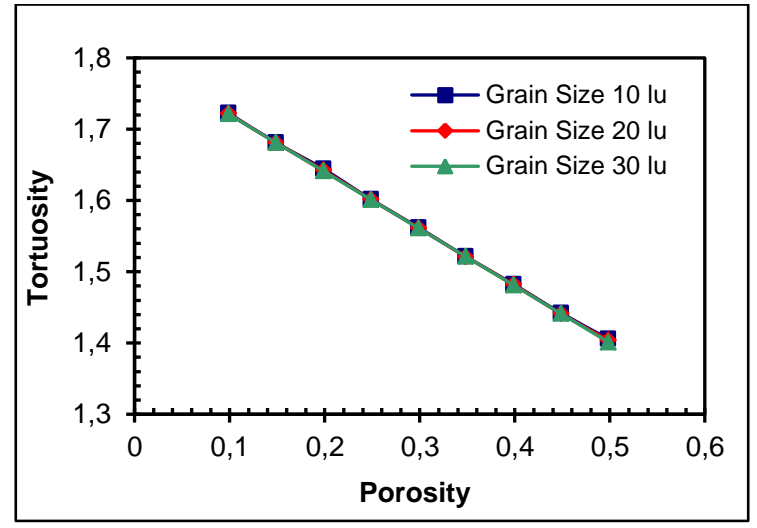

(b) Rectangular Obstacles

Figure 5. Comparison of simulated tortuosity of the porous system as a function of porosity for spherical and rectangular shaped obstacles with different grain sizes

The comparison of simulation results of tortuosity both spherical and rectangular obstacles for each grain sizes can be seen in Figure 5. It is shown that the trend lines of both obstacle shapes are equal and gives the straight lines curves. The grain sizes 10 lattice units give relative higher results of tortuosity values than grain sizes 20 lattice units and 30 lattice units. The reason is that the porous media constructed by the 10 lattice units more heterogeneous than by the others grain sizes. It is concluded that for a given obstacle configurations the tortuosity simulated with different grain sizes and shapes were found to be close to each other.

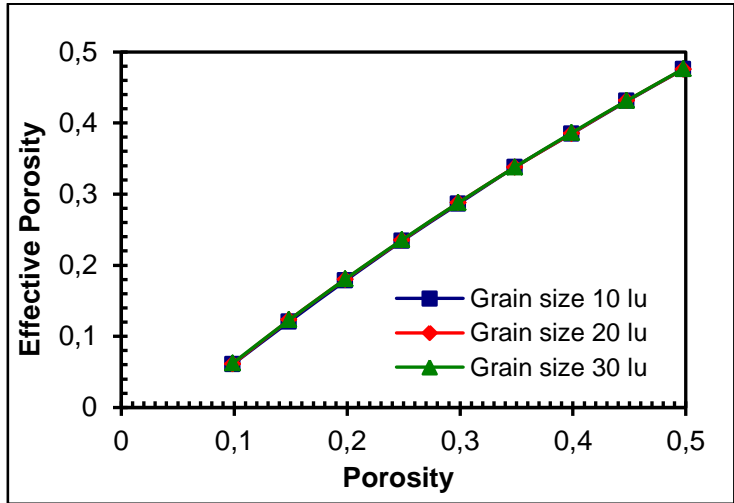

(a) Spherical Obstacles

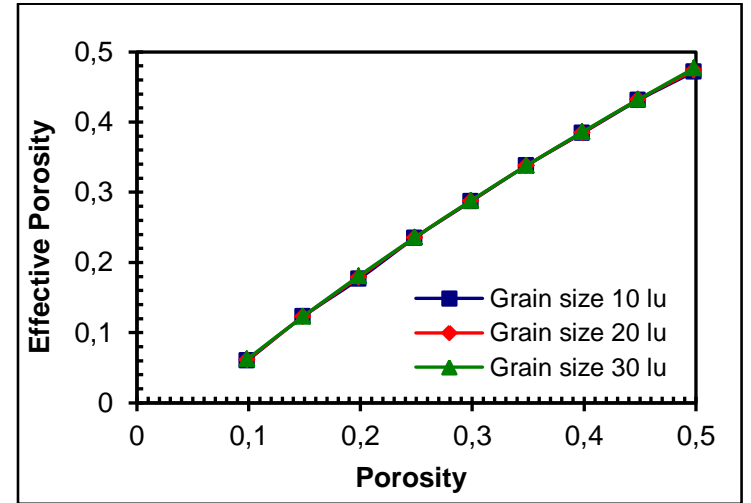

(b) Rectangular Obstacles

Figure 6. Comparison of simulated effective porosity as a function of porosity for spherical and rectangular obstacles with different grain sizes 
The comparison of simulated effective porosity of porous media as a function of porosity for both spherical and rectangular obstacles can be seen in Figure 6. It shows that even though the grain sizes and shapes of solid particles are different, but the effective porosity results for each grain size were quite similar. The effective porosity value for grain size 10 lattice units is the lowest compared to the 20 lattice units and 30 lattice units, respectively. This is due to the absolute porosity of porous media constructed by grain size 10 lattice units is also lowest than the others.

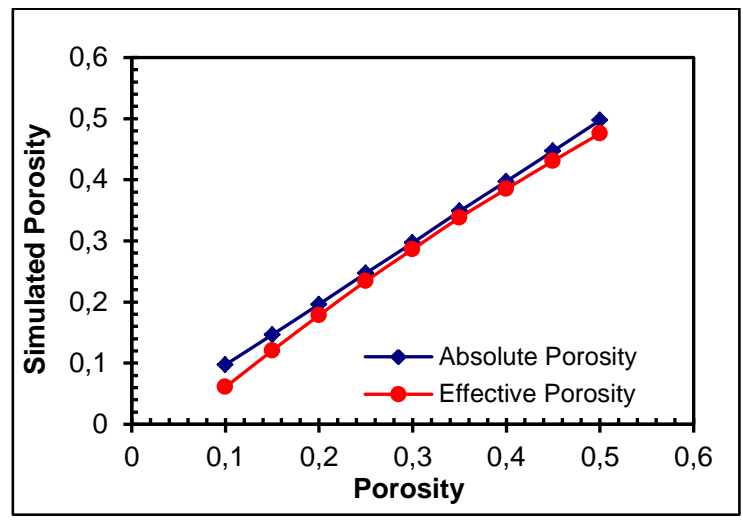

(a) Spherical Obstacles

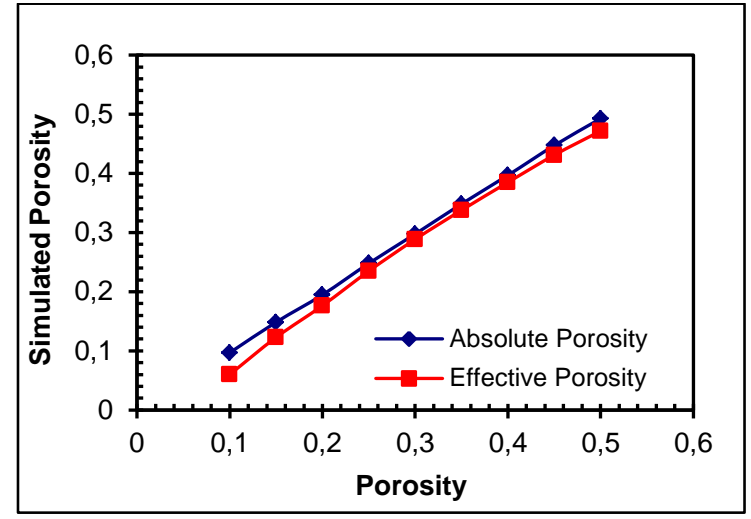

(b) Rectangular Obstacles

Figure 7. Comparison of the simulated porosity results of spherical and rectangular obstacles

From the reservoir engineering standpoints, the effective porosity results indicate a true, where commonly the effective porosity in the interconnected pores of porous media is lower than its absolute porosity. To show agreement with the simulation results, the effective porosity and absolute porosity simulated of the porous media constructed are then compared, can be seen in Figure 7. The figures were taken on the absolute porosity of the porous media constructed by the spherical and rectangular obstacles have grain size 30 lattice units.

Based on the simulation results in Figure 6 and Figure 7, it was concluded that the effective porosity is a direct function of the grain shape and size of the interconnected pores. The correlation of effective porosity of porous media as a function of absolute porosity was found. The simulated effective porosity yields a good accuracy results, and indicate a more reliable results for the heterogeneity and anisotropy of the porous media.

Permeability is the other main concern in this simulation besides effective porosity. Permeability is such an important property of porous media from the reservoir and production engineering standpoint. The importance of permeability in oil reservoir is not only in the actual volume of oil, but the rate at which the oil will flow through the reservoir. This will directly affect the economic potential of a well. Comparison of the simulated dimensionless permeability $\left(\mathrm{k} / \mathrm{Ro}^{2}\right)$ of the porous system as a function of effective porosity ( $\left.\phi_{\text {eff }}\right)$ for spherical and rectangular obstacles with different grain sizes can be seen in Figure 8.

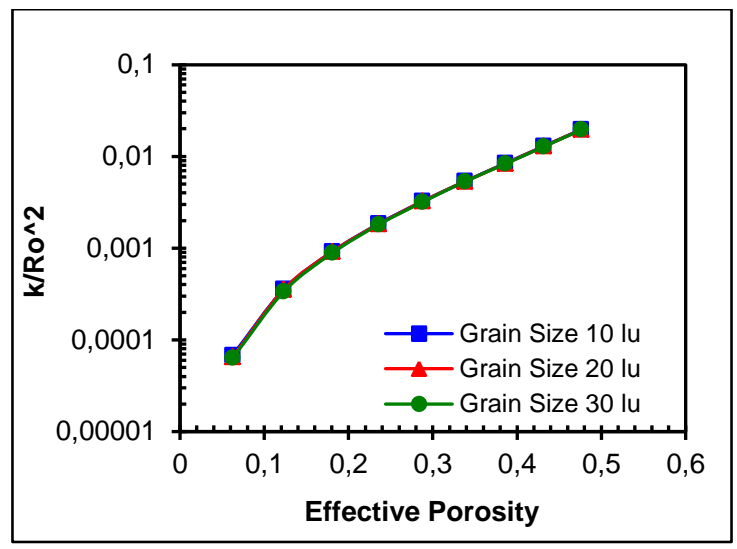

(a) Spherical Obstacles

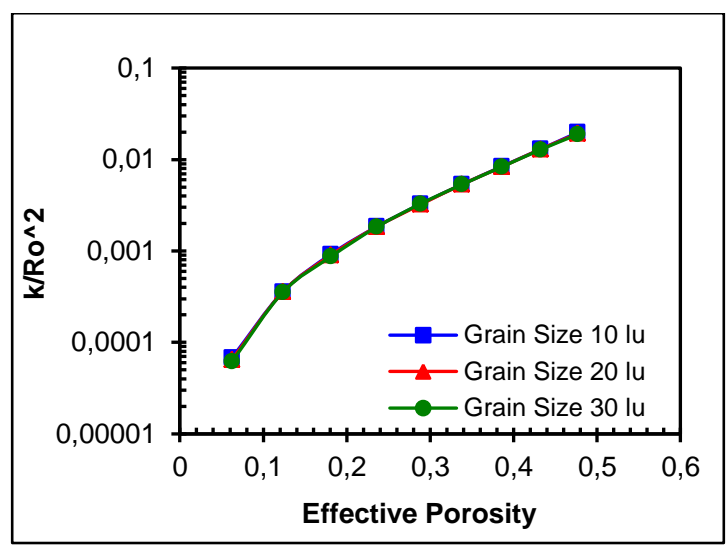

(b) Rectangular Obstacles

Figure 8. Comparison of simulated dimensionless permeability $\left(\mathrm{k} / \mathrm{Ro}^{2}\right)$ of the porous system as a function of effective porosity ( $\phi_{\text {eff }}$ ) for spherical and rectangular obstacles with different grain sizes 
The comparison of simulated dimensionless permeability $\left(\mathrm{k} / \mathrm{Ro}^{2}\right)$ of the porous system as a function of effective porosity $\left(\phi_{\text {eff }}\right)$ for spherical and rectangular obstacles with different grain sizes can be seen in Figure 8. The findings indicate that the dimensionless permeability results of both spherical and rectangular shapes for each grain sizes were quite similar. The values of dimensionless permeability of spherical obstacles were relatively higher than the values for rectangular obstacles. This is due to the fact that the effective porosity value of spherical obstacles is also higher than the one for rectangular obstacles. However, the differences in values of the permeability results of both grain shapes are relatively close. It shows that the factors in which influence effective porosity, i.e., grain size and shape, and their distributions also have a direct effect on the permeability of the porous system.

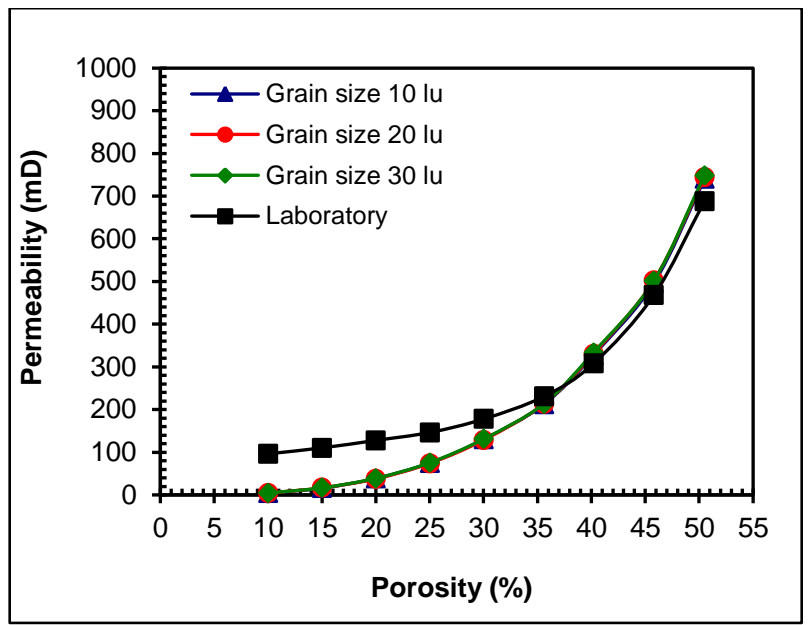

Figure 9. Comparison of the permeability results between laboratory experiment and simulation

To validate the permeability of the simulation results, a laboratory experiment was also conducted. The laboratory experiment was conducted using a core sample for different values of porosity with grain size 20-40 mesh. The comparison of permeability results by laboratory experiment and this simulation for each grain size of obstacles as a function of porosity can be seen in Figure 9. From Figure 9 it is obvious that the system is divided into two regions based on the validity of Carman-Kozeny equation used in the simulation. The results obtained in the simulation were comparable with the laboratory experimental results for that region of porosity having a value of $35 \%$ and higher. In this region, all grain size shows a good approach to those data points obtained from the laboratory experimental. However, at a lower porosity range, namely at a region of porosity lower than $35 \%$, results of the simulation were unsatisfactory compared to the laboratory experiment results. All grain sizes failed to approach the behaviour of the laboratory experimental results. It could be attributed to the fact that the Equation 11 to determine the permeability value has a validity range, and it is inapplicable for the entire range of porosity. As the permeability value is essential for simulation calculation, any deviation in the prediction of Equation 11 can lead to a large deviation in the final prediction of the simulation, which could affect its performance to give a good prediction.

The simulation results have been found to be comparable with the experimental data points for porosity ranges of $35 \%$ to higher, where the relative error does not exceed less than $10 \%$. Relative error gradually increases with decreasing values of porosity ranges lower than $35 \%$, where the average error varies from $10 \%$ to $25 \%$, respectively. It is worthy to bring to attention Equation 11 where parameters involved in this equation relate to each other. The Kozeny coefficient, $c$, is the function of tortuosity and shape factor. Surface area, as it is known has a value which is related inversely to grain size, where large grain size has a lower surface area and vice versa. Results of the simulation can be seen in Figure 9 agree with this fact through the value of permeability obtained from Equation 11. From this outcome, it could be concluded that the Carman-Kozeny equation used in this simulation to estimate permeability has a limited application that limits its validity, which depends on the porosity ranges. For low porosity ranges (less than $35 \%$ ), permeability determined from the simulation has a lower value compared with that obtained from the laboratory experiment, such an outcome was observed for all grain sizes.

However, the results from this simulation is similar with Willy's work (Smith et al., 1975) which used a capillary tube for flow network, and also Rothman's work (Rothman, 1988), where a pore-space that contains very narrow channels may produce significant errors in determining permeability if the bulk flow is dominated by narrow passageways. This problem is for practical purposes, inconsequential for flows through tubes of large radii. However, if the radius of a tube is small, then the effect of the resulting permeability could be substantial due to the quadratic dependence of permeability on the radius. The accuracy of the results is in the neighborhood of $10 \%$ to $25 \%$, with errors decreasing as the grain size increases. 
The permeability of the simulation results could be still applicable and relevant from the reservoir engineering point of view. This is because there are materials of real porous media that has a lower permeability value, especially for sandstone and limestone formations (Collins, 1976). Sandstone has a lower permeability of $5 \times 10^{-4}$ darcy $(0.5 \mathrm{mD})$, and limestone has lower permeability of $2 \times 10^{-4}$ darcy $(0.2 \mathrm{mD})$. Cole $(1969)$, has also reported on typical reservoir rocks which has a lower permeability, of $2 \mathrm{mD}$ (Bradford sandstone, Pennsylvania), $23 \mathrm{mD}$ (Upper Strawn sandstone, Texas), and $27 \mathrm{mD}$ (Bartlesville sandstone, Oklahoma). In comparison, the simulation results of permeability also have lower values of $4.39 \mathrm{mD}, 4.45 \mathrm{mD}$, and $4.61 \mathrm{mD}$ being for grain size 10 lattice unit, 20 lattice unit and 30 lattice unit, respectively.

\subsection{Displacement Efficiency}

In a displacement process, oil recovery depends on the volume of porous media or reservoir contacted by the injected fluid. A quantitative measure of this contact is the displacement efficiency $\left(E_{D}\right)$. Displacement efficiency of the displacement process in this research then could be defined as the fraction of pore volume oil displaced by the carbon dioxide as the injected fluid, or the fraction of pore volume oil that has been contacted by the carbon dioxide. Hence, it is clear that the displacement efficiency is a function of time in a displacement process. Furthermore, from this sequence of displacement efficiency, the simulation was conducted to study the effects of properties of porous media on the displacement efficiency of displacement process with particular emphasis on the effect of porosity and permeability. The simulation results of displacement efficiency, where oil is displaced by carbon dioxide in the immiscible displacement process can be seen in Figure 10 and Figure 11, each for spherical and rectangular shapes, respectively. Summary of the effective porosity and permeability effects on displacement efficiency results of spherical and rectangular shapes can be seen in Figure 12.

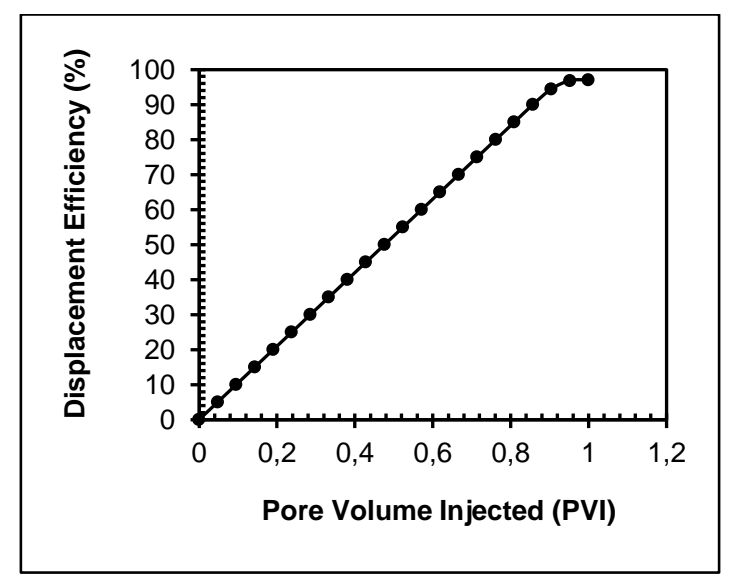

(a) Eff. Porosity $=47.56 \%$

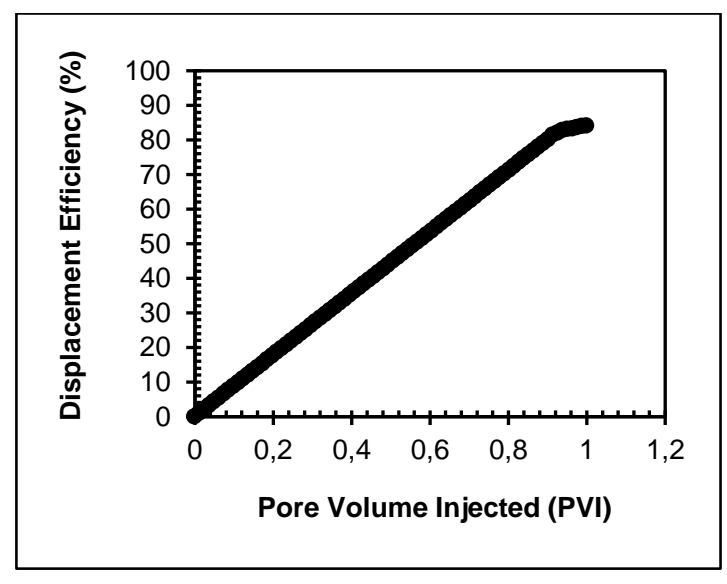

(b) Eff. Porosity $=6.126 \%$

Figure 10. Simulated displacement efficiency of spherical shapes for effective porosity of $47.56 \%$ and $6.126 \%$

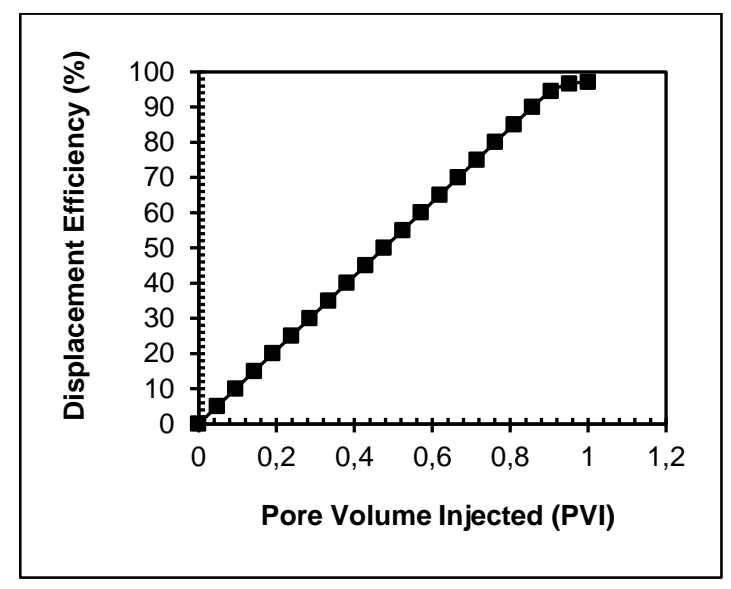

(a) Eff. Porosity $=47.173 \%$

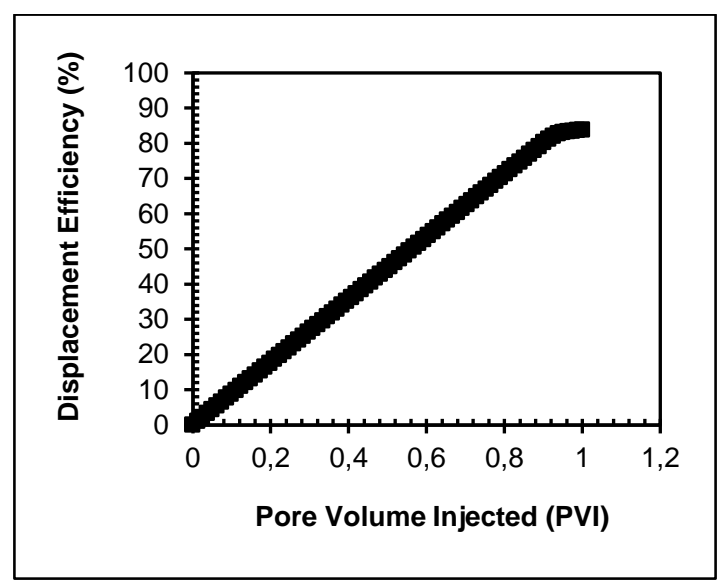

(b) Eff. Porosity $=6.001 \%$

Figure 11. Simulated displacement efficiency of rectangular shapes for effective porosity of $47.173 \%$ and $6.001 \%$ 


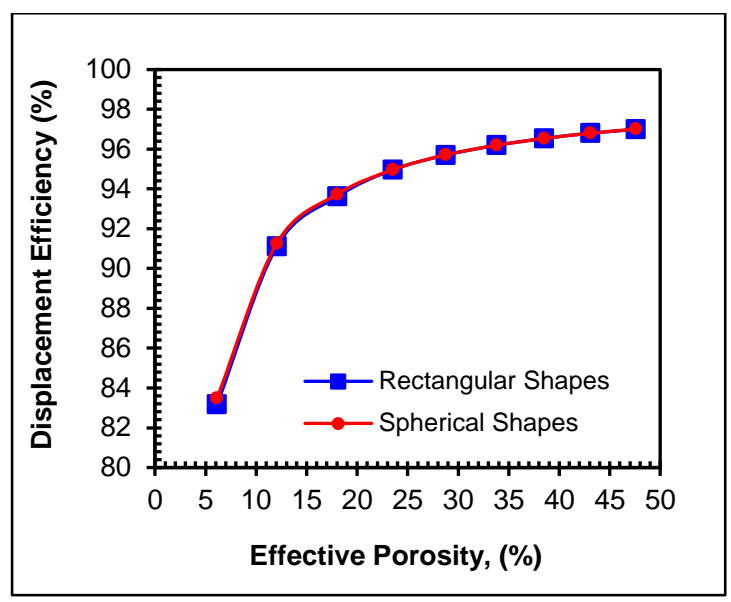

(a) Effective Porosity

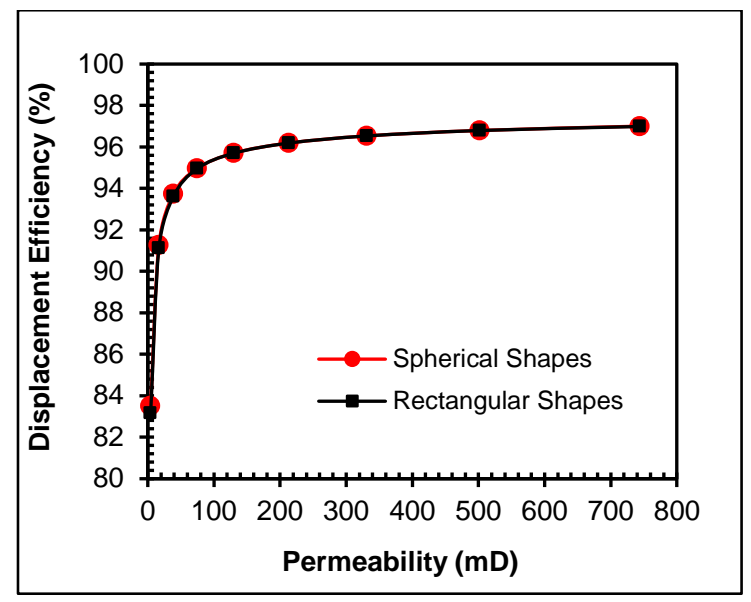

(b) Permeability

Figure 12. . Summary of simulated displacement efficiency for spherical and rectangular shapes as a function of: (a) effective porosity; and (b) permeability of porous media

From Figure 12a, it is shown that if the porous media has high values of effective porosity, the displacement efficiency that could be obtained in the displacement process would also be higher. This is true for both spherical and rectangular shapes. The trend of these curves shows a rapid increase in the value of effective porosity from $6.126 \%$ to $18.034 \%$ in spherical shapes, and $6.001 \%$ to $17.649 \%$ in rectangular shapes. The displacement efficiency value also showed an increase from $83.49233 \%$ to $93.73217 \%$ for spherical shapes and $83.16931 \%$ to $93.61726 \%$ for rectangular shapes. However, the effective porosity value above of $18.034 \%$ in spherical shapes and above $17.649 \%$ in rectangular shapes, show a relatively slow and stable increase in the displacement efficiency value. Based on the effective porosity values of the porous media, the displacement efficiency had ranges of $83.49233 \%$ to $97.00252 \%$ for spherical shapes, and $83.16931 \%$ to $96.98614 \%$ for rectangular shapes. Hence, it shows that the displacement efficiency for spherical shapes is relatively higher compared with rectangular shapes. It demonstrates that the shapes and sizes (pore geometry) and their distribution in porous media could affect the displacement efficiency.

In Figure 12b, shown the simulated displacement efficiency for spherical and rectangular shapes versus permeability of porous media is presented. It is shown that the permeability of porous media could also affect the displacement efficiency. A proportional relationship is found to exist, where, as the permeability values increase, displacement efficiency percentages also increase. Trend of the curves was quite similar to that presented in Figure 13a. However, it is worth clarifying that at permeability ranges of $4.44143 \mathrm{mD}$ to $38.83125 \mathrm{mD}$ in the spherical shapes and $4.385745 \mathrm{mD}$ to $37.95410 \mathrm{mD}$ in rectangular shapes, the curves increase rapidly after that the trend of the curves became relatively linear. It demonstrates the fact that permeability of porous media is a function of pore size and shape distribution of solid grains in the porous media. It can be concluded that permeability can affect strongly the distribution of the fluids in the pores. Hence, it was also demonstrated that changing of porous media model from low to high permeability would increase the displacement efficiency.

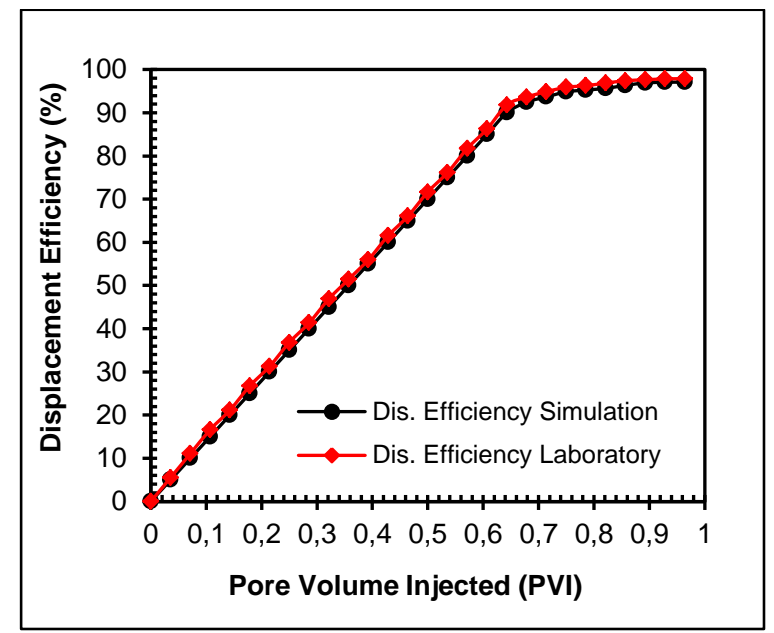

Figure 13. Comparison of displacement efficiency results from the simulation and laboratory experiments 
To validate the displacement efficiency values, which resulted from the simulation, a comparison between the simulation results and those obtained from the laboratory was made using a core model experiment. The resultsq can be seen in Figure 13. Displacement process in the laboratory was conducted with a core model having porosity of $54 \%$ and permeability of 1.28 Darcy, while for simulation the porosity was $47.56 \%$ and permeability of $744.2541 \mathrm{mD}$. From Figure 13, it can be seen that the trend of both curves is very similar, with the difference being only $0.88483 \%$. The displacement efficiency estimated result from the simulation was $97.00252 \%$, while the laboratory experiment was $97.88735 \%$. The displacement efficiency result from the laboratory experiment is higher compared with the simulation result. Based on Figure 13, it is obvious that the estimation of simulated displacement efficiency was in excellent agreement with the results of the laboratory core model experiment. The estimations closely matched of the experimental data with a difference of less than 5\%. Furthermore, it can be concluded that the estimation of displacement efficiency by this simulation model is an adequate representation of the macro-level and hence real porous media as in the laboratory experiment.

\section{CONCLUSION}

Geometry of fluid flow in a heterogeneous porous media could be simulated well by the FHP-II model of lattice gas automata. The physical models of heterogeneities constructed are heterogeneous isotropic distribution of permeability and anisotropic distribution of heterogeneous permeability. The properties of the porous media were estimated by the shape, size, number of obstacles and by the distribution of the obstacles within the volume. In determining the tortuosity, effective porosity and permeability were found to be strongly affected by grain shape and size, as well as their distribution in the porous media. However, it is obvious that to determine permeability using the Carman-Kozeny equation it is recommended to be applied to a porosity range of 35\% and higher. This is because it was found that for lower porosity range (less than 35\%) the permeability estimated was lower than obtained from the laboratory experiment.

Based on the simulation results and the discussion on estimation displacement efficiency, it is concluded that in porous media, with different values of effective porosity and permeability could affect on the displacement efficiency of the displacement process. In the porous media that has higher effective porosity and permeability values, the displacement efficiency that could be obtained in the displacement process would also be higher. Estimation of the simulated displacement efficiency was in excellent agreement with the results of the laboratory experiment, with a difference of less than $5 \%$. The estimation of displacement efficiency by this simulation model was an adequate representation of the macro-level and hence real porous media as obtained in the laboratory experiments.

\section{NOMENCLATURE}

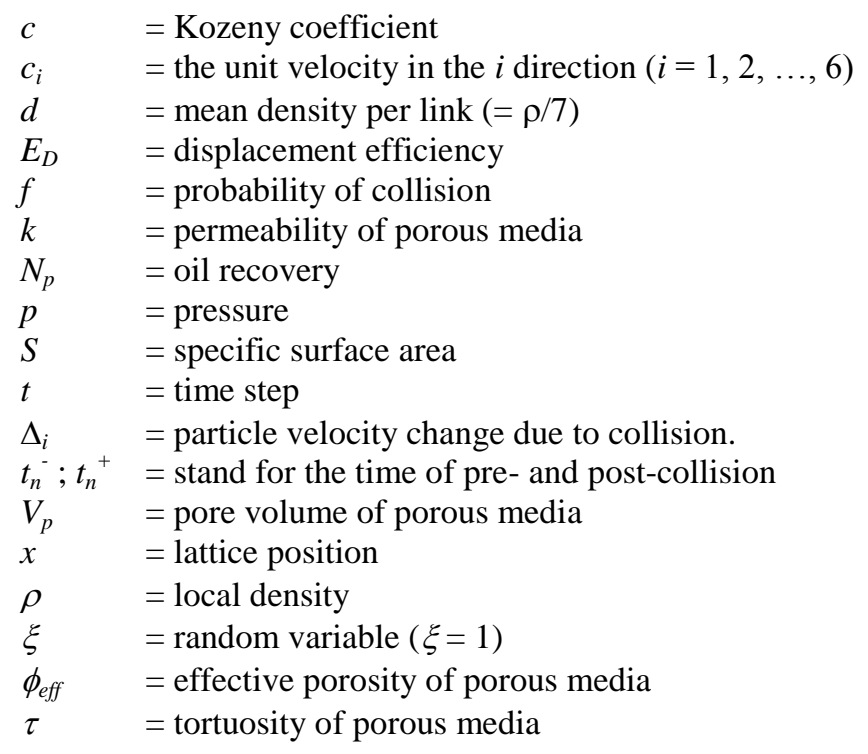

\section{ACKNOWLEDGEMENTS}

The author would like to thanks the Petroleum Engineering Department, Faculty of Mineral Technology, UPN "Veteran" Yogyakarta for the support to publish the paper. 


\section{REFERENCES}

Blunt, M. J. et al.: "Pore-level Modeling of Wetting”, Physical Review A, 46, (1995), p.7680-7699.

Blunt, M. J.: "Physically Based Network Modeling of Multiphase Flow in Intermediate-Wet Porous Media", Journal of Petroleum Science and Engineering, 14, (1997), p.1-14.

Collins, R. E.: Flow of Fluids through Porous Material, PennWell Books, Tulsa-Oklahoma.: PennWell Publishing Co., (1976), p.3-26; and 139-149.

Cole, F. W.: Reservoir Engineering Manual, Houston-Texas.: Gulf Publishing Co., (1969), p.3-39.

Dullien, F.A.L.: Porous Media: Fluid Transport and Pore Structure, Academic Press Inc., New York (1979).

Hardy, J. et al.: "Time Evolution of Two-Dimensional Model System I: Invariant States and Time Correlation Functions", Journal of Mathematical Physics, 14, (1973), p.1746-1759.

Hardy, J. et al.:"Molecular Dynamics of a Classical Lattice Gas: Transport Properties and Time Correlation Functions", Physics Review A, 13, (1976), p.1949-1961.

d' Humières, D., and Lallemand, P.: “Lattice Gas Automata for Fluid Mechanics”, Physica A, 140, (1986), p.326-335.

Frisch, U. et al.: "Lattice-Gas Automata for the Navier-Stokes Equation”, Physical Review Letters, 56, (1986), p.15051508.

Frisch, U. et al.: "Lattice Gas Hydrodynamics in Two and Three Dimensions", Complex Systems, 1, (1987), p.649-707.

Gao, Y.: "Effect of Structure on Petrophysical Properties of Porous Media." The University of Texas, Austin: Ph.D. dissertation, (1994).

Kadanoff, L. P. et al.: "From Automata to Fluid Flow: Comparison of Simulation and Theory", Physical Review A, 40, (1989), p.4527-4541.

Koponen, A. et al.: “Tortuos Flow in Porous Media”, Physical Review E, 54, (1996), p.406-410.

Koponen, A. et al.: "Permeability and Porosity of Porous Media", Physical Review E, 56, (1997), p.3319-3325.

Kristanto, D. and Awang, M.: "The Application of Lattice Gas Automata to Study Fluid Flow in a Porous Media", paper IPA01-E-006 presented at the $28^{\text {th }}$ Indonesian Petroleum Association (IPA) Annual Convention and Exhibition 2002, Jakarta, Indonesia, February 26-28.

Kristanto, D, and Awang, M.: "Estimation of Surface Tension for Two Immiscible Fluids Using Lattice Gas Automata", SPE 84892, Society of Petroleum Engineers International on Improved Oil Recovery Conference in Asia Pacific (SPE-IIORC) 2003, Kuala Lumpur, Malaysia, 20-21 October.

Larson, R. G. et al.: "Displacement of Residual Nonwetting Fluid from Porous Media", Chemical Engineering Science, 36, (1981), p.75-85.

Lee, S. H., and Chung, E. Y.: “A Cellular Automaton Model for Flow in a Reservoir", SPE Advanced Technology Series, 1, (1993), p.52-59.

Orme, M.: "Lattice Gas Methods: Fluid Dynamic from Particle Collisions", Air Filtration Review, 17, (1996), p.41-48.

Oren, P. E., and Pinczewski, W. V.: "Fluid Distribution and Pore-Scale Displacement Mechanisms in Drainage Dominated Three-phase Flow", Transport in Porous Media, 20, (1995), p.105-133.

Rothman, D. H.: “Cellular-automaton Fluids: A Model for Flow in Porous Media”, Geophysics, 53, (1988), p.509-518.

Rothman, D. H., and Zaleski, S.: Lattice Gas Cellular Automata: Simple Models of Complex Hydrodynamics, London, UK.: Cambridge University Press, (1997), p.12-60; 151-165; 203-232.

Smith, C. R.: Mechanics of Secondary Oil Recovery, Hutington-New York.: Robert E. Krieger Publishing Co., (1975), p. 36-72.

Sandrea, R., and Nielsen, R.: Dynamics of Petroleum Reservoirs Under Gas Injection, Houston, Texas.: Gulf Publishing Company, (1994), p.58-92.

Wolfram, S.: "Cellular Automaton Fluids 1: Basic Theory”, Journal of Statistical Physics, 45, (1986), p.471-529.

Zanetti, G.: "Hydrodynamics of Lattice-Gas Automata.” Physical Review A, 40, (1989), p.1539-1548.

Zaleski, S., and Appert, C.: "Lattice Gas with a Liquid-Gas Transition”, Physical Review Letters, 64, (1990), p.1-4. 